\title{
Entropy Generation Analysis of a Proton Exchange Membrane Fuel Cell (PEMFC) with a Fermat Spiral as a Flow Distributor
}

\author{
V.H. Rangel-Hernandez, C. Damian-Ascencio, D. Juarez-Robles, A. Gallegos-Muñoz, \\ A. Zaleta-Aguilar and H. Plascencia-Mora
}

Additional information is available at the end of the chapter

http://dx.doi.org/10.5772/51979

\section{Introduction}

In these last decades, fuel cells technology have emerged as an alternative to conventional electricity generation systems [1,2]. However, high operating and manufacturing costs, non-homogeneous current density production and low durability are some of the serious issues that have impeded their expansive application. Hence many research projects have been undertaken by academic and industrial sectors intended to improve the technology [3].

In this regard, some of these works have focused mainly on the optimization of the cell geometry [4-7]. An innovative design consisted of a bipolar plate with interdigitated flow field [8]. Such flow field conducts the reactants gases onto the active area and helps to eliminate the need for a separate cooling layer in the stack. Such improvement provides an increment in the performance of the PEMFC. Tuber et al. [9] proposes an optimization method based on fractal concepts to increase the performance of the bipolar plate fuel cell stacks. However, in terms of polarization curves, their performance was similar to that of a parallel channel configuration stack. The only advantage was a reduction of pressure drop. Others authors have used genetic algorithms [10,11] or biomimetical models [12] to provide better designs, maximize the net power and minimize the pressure drop. In terms of the geometry of the channel, an Archimedes spiral has been used in [13]. Here the gas flows from the center to the external part of the spiral. A reduction of the pressure drop is observed. Besides, the model shows a uniform current density production along the cell. This model considerably improves the performance of the cell.

On the contrary, other research works have focused on highlighting the major causes of inefficiencies and their location in a fuel cell $[3,14]$. The novelty of these works is that they provide crucial information such as heat and mass transfer as well as chemical and electrochemical reactions for improving the fuel cell design $[15,16]$. 
Hence the goal of this study is to identify the main sources of irreversibility of a PEM Fuel Cell by application of the Entropy Generation Method. The main difference from other fuel cell is that it uses a Fermat spiral as flow distributor. In order to have a more complete analysis of the fuel cell, a new dimensional parameter is introduced. The parameter is helpful in unveiling the effect of the mass transfer on the overall entropy generation.
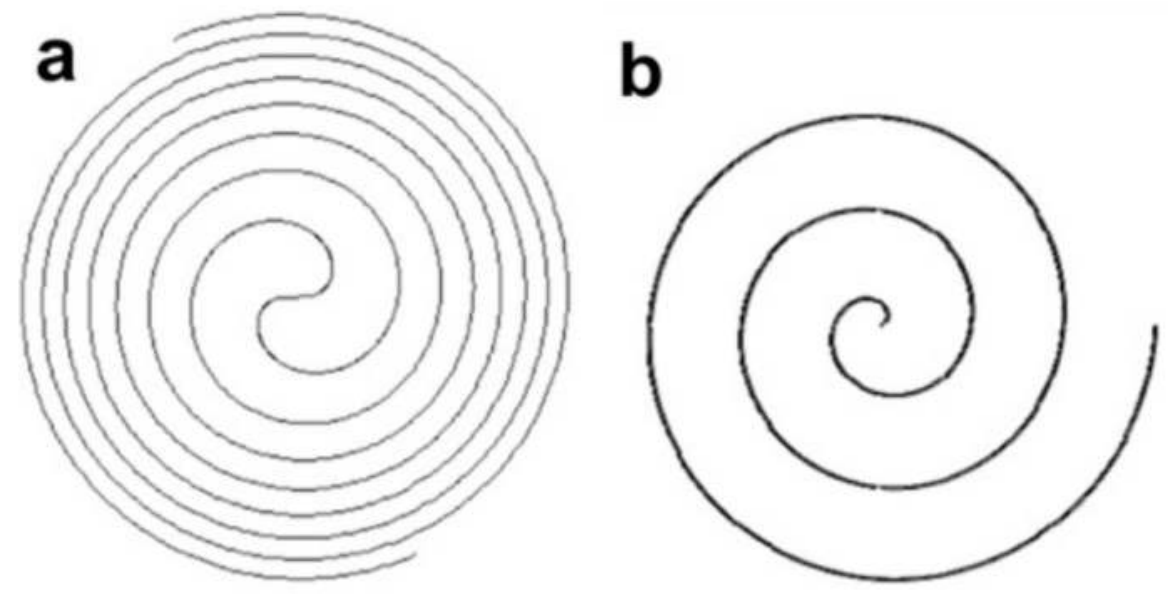

Figure 1. Spiral of (a) Fermat $r^{2}=a \theta$ (b) Archimedes $r=b \theta$.

\section{Model development}

The particular geometry of the flow channels has been thought to have larger and more uniform current densities, to avoid gas stagnation and flooding of the channels as well as to reduce pressure drops.

Fig. 1 shows the Fermat and the Archimedes spiral configuration, respectively. It is observed that the parametrical equation characterizing the Fermat spiral does not increase proportional to the angle $\theta$, which avoids generating gas flow channels of constant width. Thus four concentric Archimedes spirals have been used with a $\pi$ radian phase angle between each couple of spirals, instead of using two Fermat spirals. This permits generating two channels of constant width.

Thus, in order to fulfill the requirements of the new geometry it has been necessary to modify two zones of the fuel cell, namely, the gas flow channels and the current collectors. While the gas diffusion layers, catalyst layers, anode and cathode and the membrane have been kept constant.

The geometrical parameters are shown in Table 1. Values are similar to those used by Shimpalee and Dutta [16]. The current collectors (electrodes), gas difussion layer (GDL), catalysts layers, and the membrane have the same width of the cell.

Fig. 2 shows the three-dimensional model developed in a co- flow pattern, where the gas reactant inlets are located at the center of the spirals. The outlets are located at the external 
part of the spirals. An alternative analysis was conducted by considering the gas inlets at the outer part of the spiral and the outlets at the center of it.

Since the gas inlet is under the level of the cell, a straight circular channel has been added to consider the height of the acrylic base where the fuel cell is mounted on.

During the analysis of the model, the following assumptions were considered:

- Steady state conditions

- Non-isothermal and single phase conditions

- Isotropic materials

- Three-dimensional mass diffusion model was considered

\section{Mathematical model}

\subsection{Governing equations}

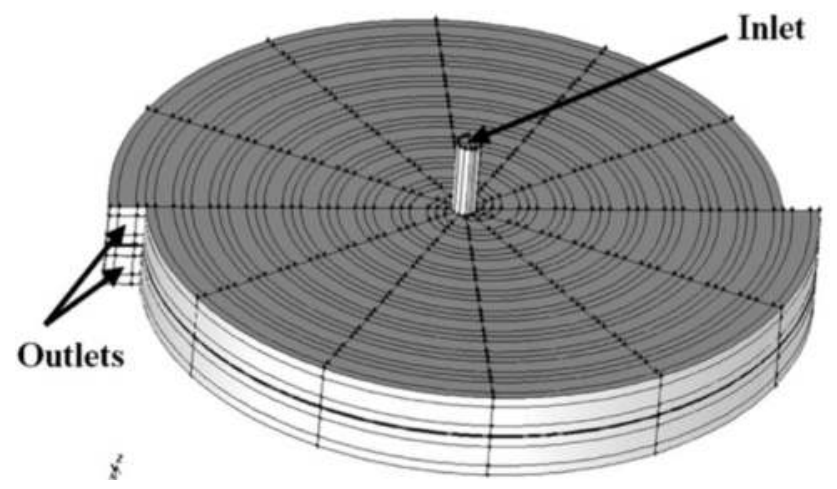

Figure 2. Computational model developed for the PEMFC with the shape of the Fermat spiral.

\begin{tabular}{ll}
\hline Side & Dimension $(\mathrm{mm})$ \\
\hline Current collector height & 1.50 \\
Channels height & 1.00 \\
Channels width & 0.80 \\
GDL height & 0.50 \\
Catalyst width & 0.03 \\
Cell width & 1.60 \\
Inlet radius & 0.53 \\
Inlet channel length & 3.50 \\
Number of turns & 4.5 \\
\hline
\end{tabular}

Table 1. Solutions to the motion equations.

The mathematical model consists of a series of partial differential equations which account for the continuity equation, the momentum equation, energy conservation and the conservation of species: 
Conservation mass

$$
\nabla \cdot(\rho \mathbf{u})=S_{i}
$$

Momentum Equations

$$
\mathbf{u} \nabla \cdot(\rho \mathbf{u})=-\nabla p+\nabla \cdot(\mu \nabla \mathbf{u})+S_{p}
$$

Hydrogen transport

$$
\nabla \cdot\left(\rho \mathbf{u} m_{H_{2}}\right)=\nabla \cdot \mathbf{J}+S_{H_{2}}
$$

Oxygen transport

$$
\nabla \cdot\left(\rho \mathbf{u} m_{\mathrm{O}_{2}}\right)=\nabla \cdot \mathbf{J}+S_{\mathrm{O}_{2}}
$$

Water transport

$$
\nabla \cdot\left(\rho \mathbf{u} m_{\mathrm{H}_{2} \mathrm{O}}\right)=\nabla \cdot \mathbf{J}+S_{\mathrm{H}_{2} \mathrm{O}}
$$

Solid phase

$$
\nabla \cdot\left(\sigma_{s o l} \nabla \phi_{s o l}\right)+S_{s o l}=0
$$

Membrane phase

$$
\nabla \cdot\left(\sigma_{m e m} \nabla \phi_{m e m}\right)+S_{m e m}=0
$$

Table 2 shows the source terms used in the calculations. From Table 2, the last equations use the following source terms:

$$
\begin{aligned}
& S_{\text {sol }}=j_{a n}^{r e f}\left(\frac{\left[H_{2}\right]}{\left[H_{2}\right]_{r e f}}\right)^{\gamma_{a n}}\left(e^{a_{a n} F \eta_{a n} / R T}-e^{-a_{c a t} F \eta_{a n} / R T}\right) \\
& S_{c a t}=j_{c a t}^{r e f}\left(\frac{\left[\mathrm{O}_{2}\right]}{\left[\mathrm{O}_{2}\right]_{\text {ref }}}\right)^{\gamma_{c a t}}\left(e^{a_{a n} F \eta_{c a t} / R T}-e^{-a_{c a t} F \eta_{c a t} / R T}\right)
\end{aligned}
$$

For Eqs. (4) and (5), $\eta$ is the electrode over-potential which results from the difference between the solid and the membrane potentials, $\phi_{\text {sol }}$ and $\phi_{m e m}$. At the anode side:

$$
\eta_{a n}=\phi_{\text {sol }}-\phi_{m e m}
$$

At the cathode side:

$$
\eta_{\text {cat }}=\phi_{\text {sol }}-\phi_{m e m}-V_{o c}
$$

Where $V_{o c}$ is the open circuit voltage of the cell and can be computed as follows:

$$
V_{o c}=0.0025 T+0.2329
$$

where, $T$ must be provided in Kelvin degrees and $V_{o c}$ in volts. The equation for the water transport coefficient, $\alpha$, is given as:

$$
\alpha(x, y)=n_{d}(x, y)-\frac{F}{I(x, y)} D_{w}(x, y) \frac{C_{w c}-C_{w a}}{t_{a}}
$$

From Eq. (13), it can be noticed that this is a function of the water activity at the anode side. The electrosmotic drag coefficient, $n_{d}$, is computed as shown in Eq. (14) and (15): 


\begin{tabular}{|c|c|c|}
\hline \multicolumn{3}{|c|}{ Equation Source terms } \\
\hline$(1)$ & $\begin{array}{l}S_{i}=S_{\mathrm{H}_{2}}+S_{\mathrm{H}_{2} \mathrm{O}} \\
S_{i}=S_{\mathrm{O}_{2}}+S_{\mathrm{H}_{2} \mathrm{O}} \\
S_{i}=0\end{array}$ & $\begin{array}{l}\text { at the anode catalyst } \\
\text { at the cathode catalyst } \\
\text { otherwise }\end{array}$ \\
\hline (2) & $\begin{array}{l}S_{p}=-\frac{\mu}{\beta} \mathbf{u} \\
S_{p}=0\end{array}$ & $\begin{array}{l}\text { in the porous region } \\
\text { otherwise }\end{array}$ \\
\hline (3) & $S_{H_{2}}=-\frac{M_{H_{2}}}{2 F} R_{a n}$ & at the anode catalyst \\
\hline (4) & $S_{\mathrm{O}_{2}}=--\frac{M_{\mathrm{O}_{2}}}{4 E} R_{\text {cat }}$ & at the cathode catalyst \\
\hline (5) & $S_{\mathrm{H}_{2} \mathrm{O}}=--\frac{M_{\mathrm{H}_{2}}=}{2 F} R_{c a}$ & at the anode/cathode catalyst \\
\hline (6) & $\begin{array}{l}S_{\text {sol }}=-S_{a n} \\
S_{\text {sol }}=+S_{c a t} \\
S_{\text {sol }}=0\end{array}$ & $\begin{array}{l}\text { at the anode catalyst } \\
\text { at the cathode catalyst } \\
\text { otherwise }\end{array}$ \\
\hline (7) & $\begin{array}{l}S_{\text {mem }}=S_{\text {an }} \\
S_{\text {mem }}=-S_{\text {cat }} \\
S_{m e m}=0\end{array}$ & $\begin{array}{l}\text { at the anode catalyst } \\
\text { at the anode catalyst } \\
\text { otherwise }\end{array}$ \\
\hline
\end{tabular}

Table 2. Solutions to the motion equations.

$$
\begin{gathered}
n_{d}(x, y)=0.0049+2.02 a_{a}-4.53 a_{a}^{2}+4.09 a_{a}^{3} \quad a \leq 1 \\
n_{d}(x, y)=1.59+0.159\left(a_{a}-1\right) \quad a>1
\end{gathered}
$$

where, $a_{a}$ is the water activity at the anode side. The water diffusion coefficient, $D_{w}$, is calculated by applying Eq. (16) [15]:

$$
D_{w}=5.5 \times 10^{-11} n_{d} e^{2416\left(\frac{1}{303}-\frac{1}{T_{s}}\right)}
$$

The concentration of water depends on both density and weight of the dry membrane as shown in Eq. (17) and (18):

$$
\begin{gathered}
C_{w k}(x, y)=\frac{\rho_{m, d r y}}{M_{m, d r y}}\left(0.043+17.8 a_{K}-39.8 a_{K}^{2}+36.0 a_{K}^{3}\right) \quad a_{k} \leq 1 \\
C_{w k}(x, y)=\frac{\rho_{m, d r y}}{M_{m, d r y}}\left(14.0+1.4\left(a_{k}-1\right)\right) \quad a_{k}>1
\end{gathered}
$$

Where the $k$ subscript stands for either the anode or the cathode, and $\rho_{m, d r y}, M_{m, d r y}$ are the density and the weight of the dry membrane, respectively. The water activity is defined as:

$$
a_{k}=\frac{X_{w, k}(x, y) P(x, y)}{P_{w, k}^{s a t}}
$$

Where $X_{w, k}$ is the molar fraction of the water, either at the anode or at the cathode side. The water saturation pressure is calculated as follows:

$$
\log _{10} P_{w, k}^{s a t}=-2.1794+0.02953(T-273.15)+10^{-5}(T-273.15)^{2}+1.4454 \times 10^{-7}(T-273.15)^{3}
$$


The membrane conductivity, $\sigma_{m e m}$ is computed by using Eq. [15]:

$$
\sigma_{\text {mem }}=\epsilon(0.514 \lambda-0.326) e^{1268\left(\frac{1}{303}-\frac{1}{T}\right)}
$$

Where $\lambda$, the water content, is calculated by applying Eq. [16]:

$$
\begin{gathered}
\lambda=0.043+17.18 a_{a}-39.85 a_{a}^{2}+36.0 a_{a}^{3} \quad a_{a} \leq 1 \\
\lambda=14.0+1.4\left(a_{a}-1\right) \quad a>1
\end{gathered}
$$

\subsection{Entropy generation equations}

In order to determine the main sources of irreversibility in a fuel cell, it is necessary to use a homogeneous thermodynamic property. In this regard, the entropy generation method is an effective tool. However the analysis needs to be seen from a local point of view, so entropy generation equations have been derived for a local application.

An expression for the local rate of entropy generation can be provided in terms of fluxes and gradients in the system, as follows:

$$
\sigma^{S}=\mathbf{q} \cdot \nabla\left(\frac{1}{T}\right)-\Sigma J_{k} \cdot\left(\frac{\mu_{k}}{T}\right)-\frac{1}{T} p^{v} \cdot \nabla v-P^{v}: \nabla v-\frac{1}{T} i \cdot \epsilon
$$

From a local point of view, Eq. (21) provides important information about the phenomenological causes of the irreversibility. The first term is the entropy generation due to heat transfer, the second due to mass transfer, the third and fourth due to mechanical dissipation and the fifth is due to ohmic losses. Strictly, the entropy generation may be expressed in terms of physical phenomena:

$$
\sigma^{S}=\sigma_{H T}+\sigma_{M F}+\sigma_{M D}+\sigma_{O h m}
$$

Thus the total entropy generation can be derived by integration of the local entropy generation, $\sigma$, in each computational domain, as follows

$$
\sigma_{T, i}=\int_{V} \sigma_{i} d V
$$

In many local rate entropy generation applications is usual to involve dimensionless parameters in order to indicate the dominant phenomenon in a system [17]. However such dimensionless parameters involve only heat transfer and fluid friction phenomena. For example, the most used is the Bejan number (Be) [17]. So when the entropy generation is due only to mechanical dissipation and heat transfer, i.e. $B e>>1 / 2$, this dimensionless parameter indicates that heat transfer irreversibilities are dominant. In particular, however, in fuel cells the entropy generation not only depends on heat transfer and fluid friction but only on mass diffusion. In view of this discussion, a new dimensionless parameter is proposed in Eq. (27) [13]:

$$
\Pi=\frac{\sigma_{M F}}{\sigma_{S}}
$$


Accordingly, $\Pi$ expresses the ratio of the entropy generation due to mass diffusion to the total entropy production. As mass diffusion effects become insignificant, i.e. $\Pi<<1=3$, the entropy distribution is completely expressed.

\section{Boundary conditions}

\subsection{Cell operating parameters}

The analysis considers that the fuel cell is operating at a pressure of $101.325 \mathrm{kPa}$ and a temperature of $343 \mathrm{~K}$. The open circuit voltage at this temperature is assumed to be $V_{o c}=$ $1.0904 \mathrm{~V}$. The gas inlet condition is defined as the cell velocity inlet. The gas outlet condition boundary was defined as the cell pressure outlet, with a gauge pressure of zero, i.e. gases are exhausted at atmospheric pressure. The inlet conditions of the gases, velocity and humidity are listed in Table 3.

\begin{tabular}{l|cccc}
\hline & VLH & LH & HH & VHH \\
\hline Anode & & & & \\
Velocity $(m / s)$ & 1.7325 & 1.830 & 2.210 & 2.560 \\
$y_{\mathrm{H}_{2}}$ & 0.7286 & 0.6367 & 0.4073 & 0.2962 \\
$y_{\mathrm{H}_{2} \mathrm{O}}$ & 0.2713 & 0.3632 & 0.5926 & 0.7037 \\
Cathode & & & & \\
Velocity $\mathrm{m} / \mathrm{s}$ & 7.330 & 7.910 & 9.050 & 12.90 \\
$y_{\mathrm{O}_{2}}$ & 0.3020 & 0.2880 & 0.2637 & 0.2085 \\
$y_{\mathrm{H}_{2} \mathrm{O}}$ & 0.6489 & 0.6178 & 0.5694 & 0.4384 \\
$y_{\mathrm{N}_{2}}$ & 0.0489 & 0.0940 & 0.1667 & 0.3528 \\
\hline
\end{tabular}

Table 3. Solutions to the motion equations.

As for the humidity conditions, these have been labeled as very high humidity (VHH), high humidity $(\mathrm{HH})$, very low humidity $(\mathrm{VLH})$ and low humidity $(\mathrm{LH})$ according to Dutta et al. [16].

\subsection{Boundary conditions at the current collector}

The external faces of the current collectors are regarded as walls at constant electrode potential, i.e. potentiostatic cell. Thus the electric potential at the anode side is $V_{\text {sol }}=0$ and at the cathode side is $V_{\text {sol }}=V_{\text {cell }}$.

\subsection{Boundary conditions at the interface between channel and porous media}

The interfaces between the gas flow channels and the GDL (and between the diffuser and the catalyst layer) are considered as porous media with a permeability of $\beta=2 \times 10^{-10} \mathrm{~m}^{2}$ and a porosity of $\epsilon=0.7$.

The reference current density for the anode and cathode sides are, respectively,

$$
j_{a}^{r e f}=2 \times 10^{9} \mathrm{~A} / \mathrm{m}^{3}, \quad j_{c}^{r e f}=4 \times 10^{6} \mathrm{~A} / \mathrm{m}^{3}
$$




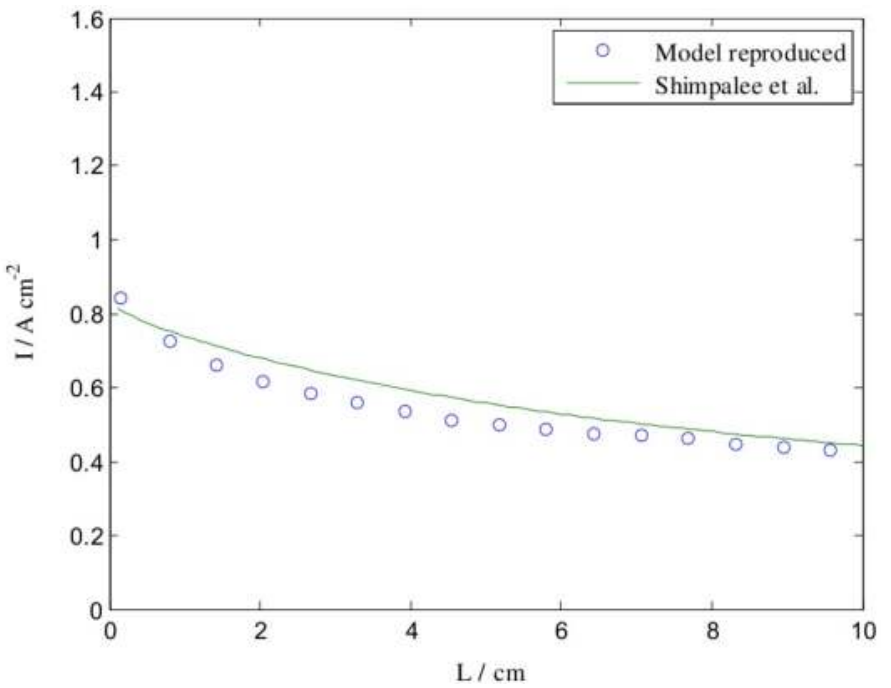

Figure 3. Computational model developed for the PEMFC with the shape of the Fermat spiral. And the reference diffusivities are,

$$
\begin{aligned}
& D_{H_{2}}=6 \times 10^{-5} \mathrm{~m}^{2} / \mathrm{s}, \quad D_{\mathrm{O}_{2}}=3.5 \times 10^{-5} \mathrm{~m}^{2} / \mathrm{s} \\
& D_{\mathrm{H}_{2} \mathrm{O}}=6 \times 10^{-5} \mathrm{~m}^{2} / \mathrm{s}, \quad D_{\mathrm{N}_{2}}=8 \times 10^{-5} \mathrm{~m}^{2} / \mathrm{s}
\end{aligned}
$$

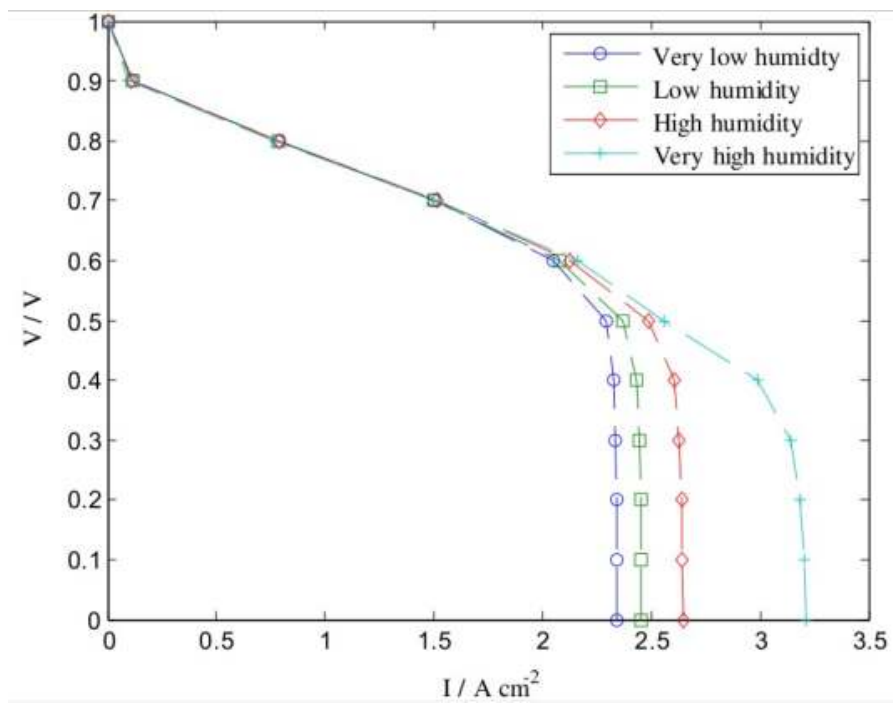

Figure 4. Computational model developed for the PEMFC with the shape of the Fermat spiral. 


\section{Validation of the model}

The analysis of the model presented in the previous sections considers three cases. Case 1 , analyzes the behavior of the cell with the velocities and mass fractions listed in Table 3. Besides, this case considers the gas inlets at the center of the spiral and the outlets at the external side of the spiral. In Case 2, the velocity inlets are increased twofold, i.e. mass flow is doubled, and the rest of the conditions remain unchanged. In Case 3, the inlets and outlets are reversed, i.e. the inlet gases are located on the external side of the spiral and the outlet gases at the center. The inlet conditions for Case 3 remain as in Table 3. Case 1 and 2 are analyzed considering four humidity conditions, while Case 3 is analyzed considering two conditions only, VHH and VLH.

The governing equations are solved with a specialized code that is based on the finite volume technique, and the application of an added module. The SIMPLE algorithm is used in the solution of the governing equations in a segregated form. This algorithm uses a relationship between velocity and pressure corrections to enforce mass conservation and to obtain the pressure path using the following steps: first the NaviereStokes equations are solved in the $\mathrm{x}, \mathrm{y}$ and $\mathrm{z}$ direction; then, a pressure correction equation is used to enforce a mass balance; next, the species equations are solved with the data obtained in the previous steps; finally, the potential fields are solved.

The model validation has been performed by comparing the results with those provided by Dutta et al. [16] for the current distribution in a straight channel. Fig. 3 shows the comparison between both models. The results of Dutta and Shimpalee range from $0.8 \mathrm{~A} / \mathrm{cm}^{2}$ at the entrance of the channels to about $0.42 \mathrm{~A} / \mathrm{cm}^{2}$ at the exit of them, whereas the present model reports values ranging from $0.81 \mathrm{~A} / \mathrm{cm}^{2}$ at the inlet and to $0.41 \mathrm{~A} / \mathrm{cm}^{2}$ at the outlet of the channel.

\section{Analysis of results}

Fig. 4 shows the polarization curves for each one of the humidity conditions proposed in the present paper. It can be observed that the dependence of the cell voltage on the humidity concentrations and on the current density is strongly nonlinear. Noteworthy that as the humidity increases the maximum current density so does. The slight slope present at low values of the current density in the polarization curves indicates minor ohmic losses, however, at high values of the current density the polarization curves fall quickly as a consequence of higher concentration losses. For the case of very high humidity condition, the polarization curve extends significantly.

Fig. 5 shows the power density and entropy production curves at different humidity conditions. As observed, the left y-axis indicates the entropy generation, whereas the right $y$-axis the power density. It is evident that at low current density values, i.e. $<2 \mathrm{~A} / \mathrm{cm}^{2}$, the entropy generation rate is insignificant. In fact, as current density goes down to values below 1.5 , the entropy generation rate is practically constant. This results from the fact that activation losses are not predominant in this zone.

On the contrary, in the zone where the concentration losses are predominant, i.e. at higher current density values, the entropy production rate sharply increases. This demonstrates that the larger irreversibilities are mostly associated with the concentration losses. 

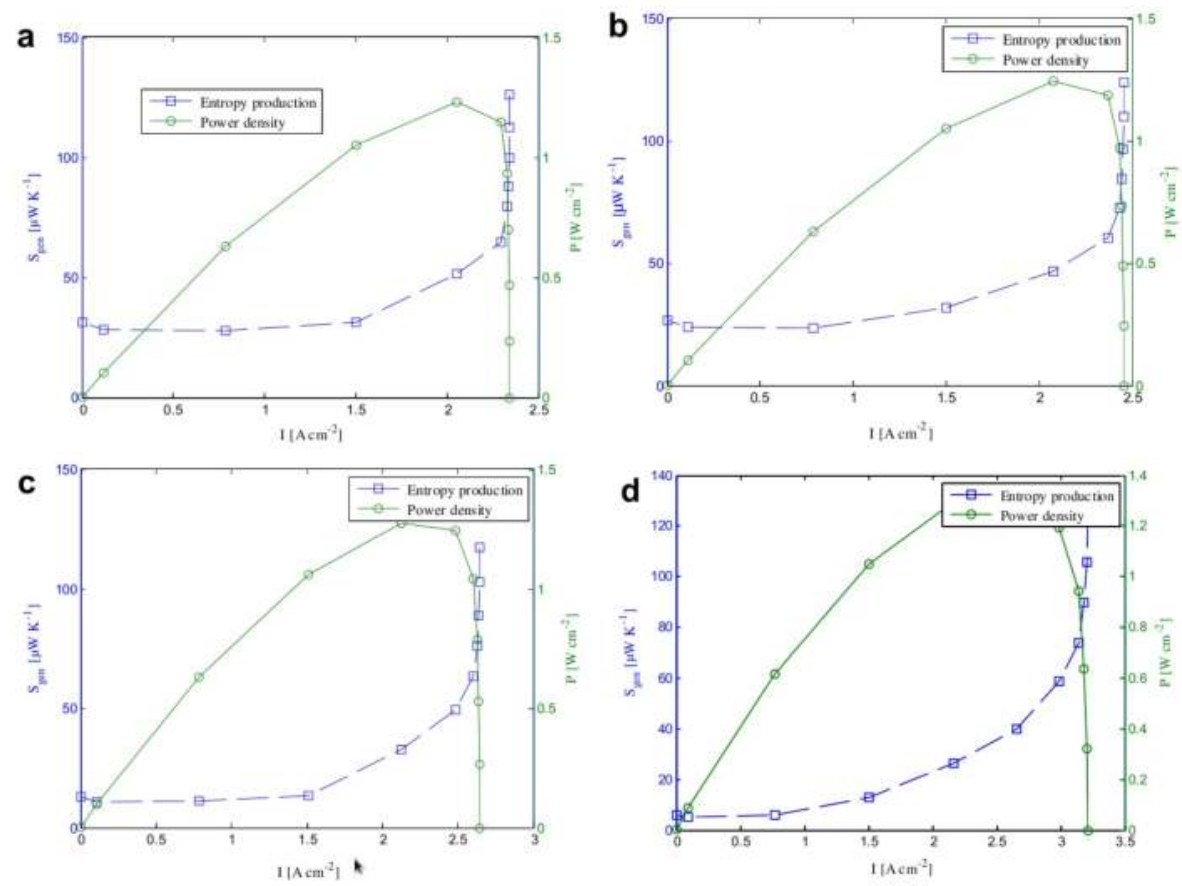

Figure 5. Power produced and entropy production for a) very low b) low c) high and d) very high humidity condition.

An important characteristic in the design of fuel cells has to do with the broadening of the power density zone as long as possible. This can only be achieved by increasing the humidity concentration as can be inferred from Fig. 5(a-d). In fact, it can be observed that the highest power density is roughly $1.25 \mathrm{~W} / \mathrm{cm}^{2}$ at current density of roughly $2.1 \mathrm{~A} / \mathrm{cm}^{2}$ for the cases when the humidity is not so high (i.e. very low, low and high humidity conditions). For the particular case of very high humidity condition, it can be observed that the domain for the highest power density values stretches consider- ably, which can be explained due to the low entropy production rate at very high humidity conditions.

The entropy generation technique points that the lesser the entropy production rate, the better the thermodynamic performance of a system. Accordingly, it is implied that the optimal flow conditions are high humidity conditions (Fig. 2). In this flow condition, the entropy generation rate is low at any current density. As aforementioned, different sources of losses in a fuel cell leads to different distributions of entropy generation, hence a more detailed analysis is required.

Graphs in Fig. 6(a-d) provide the effect of the humidity condition on the Bejan number and the entropy production ratio proposed in this paper (i.e. П). It is important to recall that this dimensionless parameter was proposed in order to have a more complete representation of the irreversibilities present in a fuel cell. So it can be inferred that the main contribution to the entropy generation rate is obtained when the gases are mixed, since it is practically impossible 
for the reverse process of separation to occur spontaneously. The second term in Eq. (21) demonstrates that the entropy generation rate due to mass transfer depends proportionally on the diffusion coefficient, the concentration and the chemical potential, but inversely on the temperature. Thus it can be implied that the dependence of these irreversibilities on the temperature is high.

From Fig. 6(a-d) it can be observed that the concentration losses decrease as the current density increase. In fuel cells the reaction temperature remains nearly constant at high current densities but the total entropy generated increase asymptotically (as demonstrated by the Bejan number), therefore the entropy generated due to species diminishes quickly.
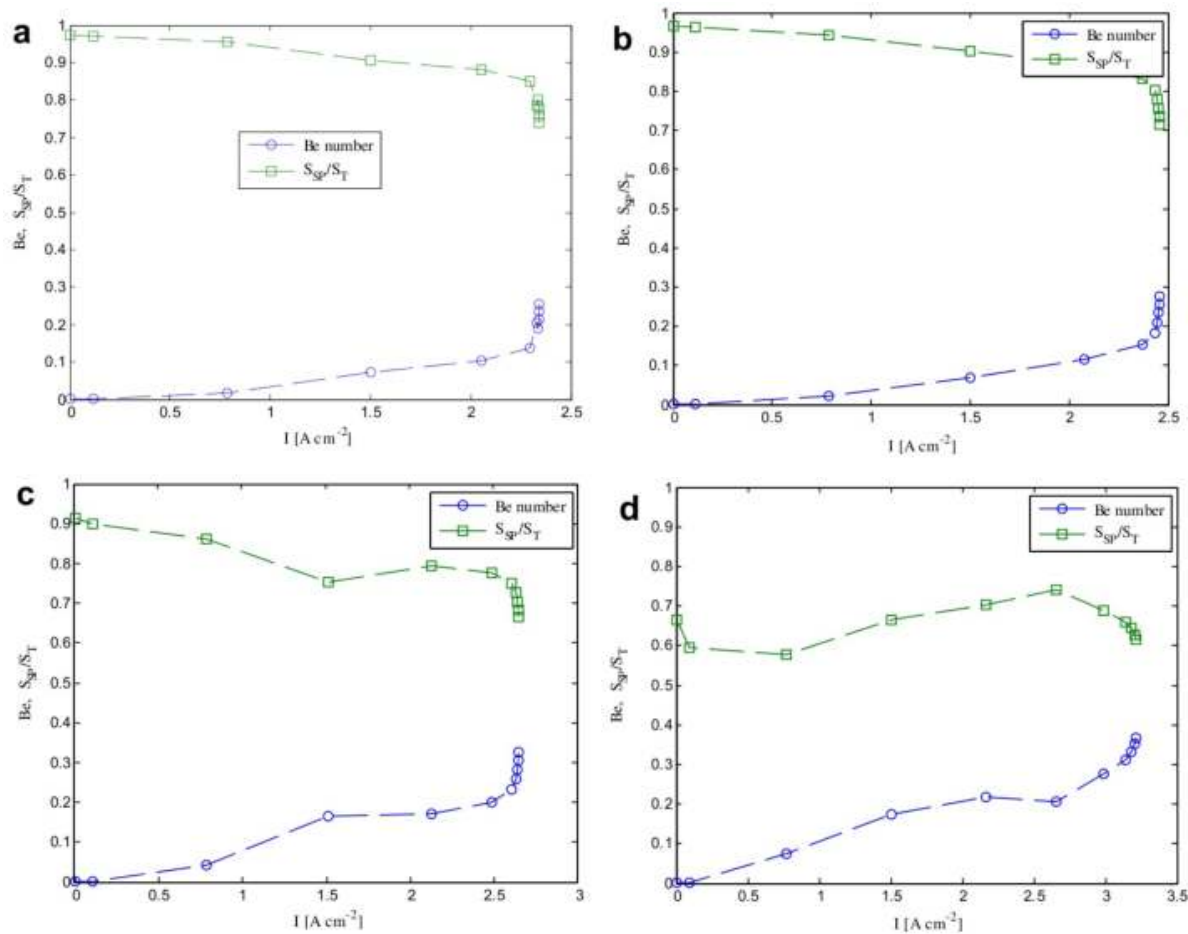

Figure 6. Entropy distribution for a) very low b) low c) high and d) very high humidity condition.

The entropy generation due to heat transfer depends on the temperature gradient (refer to Eq. (21)). Then because of a higher rate reaction in the anode, the current density and the temperature gradient increase, thereby conducting to a more generation entropy. Likewise at higher values of humidity the entropy generation due to heat transfer increases, Fig. 6 . As mentioned above, this source of entropy generation depends directly on the temperature gradient, and an increment in humidity improves the conductivity in the cell. So, heat transfer losses become more relevant.

Finally, Fig. 7 shows the entropy distribution due to ohmic losses. As the current density increases, the entropy generation so does. This is the result of the reaction rate occurring in 
the membrane. This reaction diminishes the surface area where it occurs and an elec- trical resistance is produced. Then the ohmic losses are reduced as long as the humidity conditions are increased. Such behavior comes from the fact that at higher humidity conditions the amount of water on the electrodes improves the conductivity.

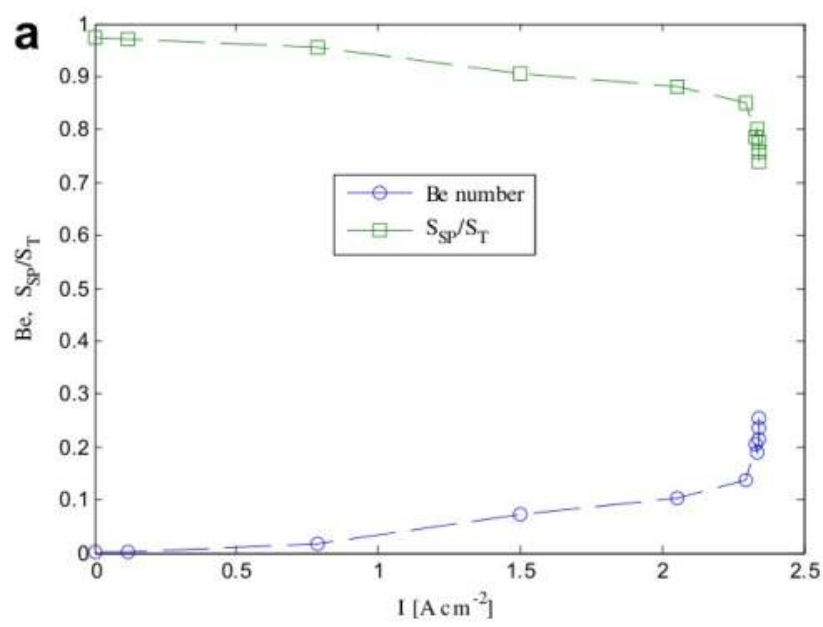

Figure 7. Entropy distribution for ohmic losses.

\section{Conclusions}

The approach of the entropy generation has been used in order to identify the main sources of irreversibility in a proton exchange membrane fuel cell. It has been shown that through a dimensionless parameters it is easier to identify the phenomenon that most influence on the irreversibility sources.

The analysis presented in this Chapter has turned out to be somewhat ambivalent since the lowest values of entropy generation are present at very low humidity conditions, whereas at high humidity conditions the entropy generation is high but, at this point, the highest density production is reached. In designing a fuel cell it comes to think what is better: a technical improvement or an economical minimization.

As for the activation losses, they remain steady for all of the cases studied because of the same operating conditions. The ohmic losses are similar too, since the properties of the material of the fuel cell do not change when the humidity is varied. In the case of the water distribution, its effect on the concentration losses is clearly noticeable.

The overall analysis demonstrates that the main sources of irreversibility in a fuel cell are the concentration losses for the most part of the operational domain. Contrarily, the irreversibilities due to the heat transfer phenomena are not dominant. As for the acti- vation losses, it is important to mention that they were not analyzed since they are already included in the concentration losses. 
Finally, a new dimensionless parameter is proposed herein. The dimensionless parameter is defined as the ratio of entropy production due to mass transfer to the total entropy production as shown in Eq. (24). The order of magnitude of the Bejan number is lower than the ratio of entropy production. For this reason, it is recommended to use this ratio to characterize fuel cells along with the Bejan number.

The use of this new dimensionless parameter can be further investigated as well as the approach presented here to perform a more complete entropy generation analysis.

\section{Acknowledgements}

The authors thank to CONACYT for the partial support in the development of this work.

\section{Author details}

Rangel-Hernandez V. H.

Mechanical Engineering Department, Engineering Division, University of Guanajuato, Palo Blanco $S / N, 36885$, Salamanca, Mexico

Damian-Ascencio C. E. Physics Department, Sciences Division, University of Guanajuato, C.P. 37150, León, Mexico

\section{References}

[1] Anonymous (2004) Fuel cell handbook, 7th ed. Morgantown: US Department of Energy, National Energy Technology Laboratory.

[2] Massardo AF, Lubelli F (2000) Internal reforming solid oxide fuel cell-gas turbine combined cycles (IRSOFC-GT): part A- cell model and cycle thermodynamic analysis. J Engg Gas Turbines Power 122: 27-35.

[3] Sciacovelli A, Verda V (2009) Entropy generation analysis in a monolithic-type solid oxide fuel cell (SOFC). Energy 34:850-865.

[4] Nguyen PT, Berning T, Djilali N (2004) Computational model of a PEM fuel cell with serpentine gas flow channels. J Power Sourc 130:149-157.

[5] Kazim A, Liu HT, Forges P. (1999) Modeling of performance of PEM fuel cells with conventional and interdigitated flow fields. J Appl Electrochem 29:1409-1416.

[6] Birgersson E, Vynnycky MA (2006) Quantitative study of the effect of flow- distributor geometry in the cathode of a PEM fuel cell. J Power Sourc 153:76-88.

[7] Li Xianguo, Sabir Imran (2005) Review of bipolar plates in PEM fuel cells: flow-field designs. Int J Hydrogen Energ 30:359-371.

[8] Chow CY, Wozniczka B, Chan JK (1999) Integrated reactant and collant fluid flow field layer for a fuel cell with membrane electrode assembly. Canadian Patent No. 2,274,974.

[9] T?ber K, Oedegaard A, Hermann M, Hebling C. (2004) Investigation of fractal flowfields in portable proton exchange membrane and direct methanol fuel cells. J Power Sourc. 31:175-181.

[10] Senn SM, Poulikakos D (2004) Tree network channels as fluid distributors constructing double-staircase polymer electrolyte fuel cells. J A Phys. 96:842-852. 
[11] Senn SM, Poulikakos D.(2006) Pyramidal direct methanol cells. Int J Heat Mass Tran. 49:1516-1528.

[12] Chapman A, Mellor I. (2003) Development of biomimetic flow field plates for PEM fuel cells. Eighth grove fuel cell symposium, September, London, UK.

[13] Damian-Ascencio C E, Hern?ndez-Guerrero A, Ascencio-Cendejas F, Juarez- Robles D (2010) Entropy generation analysis for a PEM fuel-cell with a biomimetic flow field, IMECE, Lake Buena Vista, Florida U.S.

[14] Alvarez T, Valero A, Montes JM (2006) Thermoeconomic analysis of a fuel cell hybrid power system from the fuel cell experimental data. Energy 31:1358-1370.

[15] Springer TE, Zawodzinski TA, Gottesfeld S.(1991) Polymer electrolyte fuel cell model. J Electrochem Soc. 138:2334-2341.

[16] Dutta S, Shimpalee S, Van Zee JW. (2000) Three-dimensional numerical simulation of straight channel PEM fuel cells. J Appl Electrochem. 30:135-146.

[17] Bejan (1996) A. Entropy generation minimization. New York: CRC Press. 